\title{
Influence of Boric Acid and Sucrose on the Germination and Growth of Areca Pollen
}

\section{Liyun Liu, Liyun Huang, Yan Li}

Hainan Key Laboratory of Tropical Oil Crops Biology/Coconut Research Institute, Chinese Academy of Tropical Agricultural Sciences, Wenchang, China.

Email: 1ly1995@126.com

Received May $24^{\text {th }}, 2013$; revised June $24^{\text {th }}, 2013$; accepted July $15^{\text {th }}, 2013$

Copyright (C) 2013 Liyun Liu et al. This is an open access article distributed under the Creative Commons Attribution License, which permits unrestricted use, distribution, and reproduction in any medium, provided the original work is properly cited.

\begin{abstract}
This study investigates the effect of boric acid and sucrose on the germination and growth of Areca catechu L (areca palm). Using areca pollen from Hainan, China, this study determines the influence of culture media with different concentrations of boric acid and sucrose on the germination and growth of areca pollen. Results show that the germination and growth of the pollen tube growth are highest under a boric acid concentration of $0.4 \mathrm{~g} / \mathrm{L}$ to $0.6 \mathrm{~g} / \mathrm{L}$. The germination rate of areca pollen is highest in solid medium with a sucrose concentration of $40 \mathrm{~g} / \mathrm{L}$. The optimum culture medium for developing areca pollen comprises $5 \mathrm{~g} / \mathrm{L}$ agar, $0.4 \mathrm{~g} / \mathrm{L}$ to $0.6 \mathrm{~g} / \mathrm{L}$ boric acid, $40 \mathrm{~g} / \mathrm{L}$ sucrose, and water. Furthermore, areca pollen grows most rapidly approximately $1.5 \mathrm{~h}$ after in vitro cultivation at normal temperature, and growth stabilizes at approximately $3 \mathrm{~h}$. The principle that governs the development of areca pollen is also discussed.
\end{abstract}

Keywords: Boric Acid; Sucrose; Areca Nut; Pollen; Germination

\section{Introduction}

Areca palm, also known as "lazy tree" and "money tree", is an important farming product in Hainan province, China. Approximately 400 - 600 million people worldwide chew areca nut, demonstrating the promising market prospects presented by the harvest and processing of areca nut.

Areca palm is a cross-pollinated crop, and its florescence lasts from May to July, during which Hainan experiences high temperatures and frequent rains. These adverse weather conditions can easily induce harmful flower and fruit drop from areca palm, yet related preventive measures have not been established. Previous studies have suggested that appropriate concentrations of sucrose and boron can promote the germination and growth of the pollen tube of areca pollen cultured in vitro $[1,2]$. Moreover, boron is one of the essential micronutrients in higher plants. Boron-deficient plants are characterized by dysplasia of the reproductive organs [3]. Boron deficiency in rape, for example, causes floral organ dysplasia or morphological abnormalities in the plant $[3,4]$, thereby preventing pollination. Under low boron stress, the vitality and respiratory strength of pollen decrease $[4,5]$. By in vitro areca pollen germination and growth test, this study investigates the influence of boric acid and sucrose on the germination rate of areca pollen, as well as on the duration of pollen development and germination. This research also provides theoretical basis for concentration tests on boric acid and sucrose through spraying in areca palm plantations.

\section{Materials and Methods}

\subsection{Materials}

From July to August, pollen samples were collected from the Arecanut Germplasm Resource Nursery of Coconut Research Institute of Chinese Academic of Tropical Agricultural Sciences. Native adult Hainan areca palm trees that were planted in the field six years ago were selected. The plants are characterized by consistent agronomic traits and medium growth. When the spathes fell off and male flowers opened to about one-third of full bloom, we cut a few flower branches.

\subsection{Methods}

\subsubsection{Experimental Design}

Before normal tests were conducted, several prophase preparation tests were performed with reference to the 
pollen culture medium formulas of other crops. The normal tests were designed to achieve five goals. First, the effects of culture media with different boric acid concentrations on the germination rate of areca pollen were determined. In this test, a randomized block experiment with 11 different concentration treatments and four repetitions was set. Second, the effects of culture media with different boric acid concentrations on the growth of areca pollen tube were investigated. The treatments and repetitions in this test were the same as those in the first test. Third, the effects of different sucrose concentrations on the germination rate of areca pollen were examined. This test included nine different concentration treatments and four repetitions. Fourth, the effects of different culture media on the germination rate of areca pollen were determined. Two treatments and 27 sets of matching experimental studies were carried out. Finally, the effects of different culture times on the growth of areca pollen tube were investigated. Areca pollens samples were observed every $30 \mathrm{~min}$ during culture and measured at 11 time points.

\subsubsection{Observation and Statistical Analysis}

The tests for each group were performed at room temperature (approximately $30^{\circ} \mathrm{C}$ ). The pollen grains used in each treatment with a consideration for the five goals previously mentioned were chosen from the fresh inflorescences of the same bunch on the same day. All the tests were completed on the same day to eliminate the differences caused by non-experimental treatment factors. The experiments were conducted as follows. Absorbent paper was placed in a culture dish and wetted by distilled water. Then, a gel dropper was used to extract culture medium, after which two drops of the medium were placed on each glass slide. Areca pollen grains were picked using an inoculating needle. Pollen grains were transferred onto the culture medium by gently flicking the inoculating needle. The glass slides were placed into covered culture dishes, and germination time was recorded.

A Leica DM2500 optical microscope was used to observe the areca pollen grains and capture images during the culture. Each view spans approximately 30 areca pollen grains. When pollen tube length reaches the diameter of areca pollen, the sample is defined as a germinated areca pollen. The germination ratio of areca pollen is defined as follows:

Areca pollen germination rate $(\%)=($ germinated areca pollen grain number/total areca pollen grain number) $\times$ $100 \%$.

Leica Application Suite software was used to measure the length of areca pollen tube. Excel, DPS, and ORIGIN 8.0 statistical software were used in data processing, mapping, significant difference comparison, and relevant regressions. Figures 1 and $\mathbf{2}$ were the initial germination and mature growth of areca pollen on culture media.

\section{Results}

\subsection{Effects of Boric Acid Concentration on the Development of Areca Pollen Tube}

\subsubsection{Effects of Boric Acid Concentration on Pollen Germination Rate}

Areca pollen grains were cultured on culture media with $40 \mathrm{~g} / \mathrm{L}$ sucrose, $5 \mathrm{~g} / \mathrm{L}$ agar, and boric acid concentrations of $0,0.1,0.2,0.4,0.6,0.8,1.0,2.0,3.0,4.0$, and $5.0 \mathrm{~g} / \mathrm{L}$. Each treatment was prepared with two glass slides, and each glass slide contained culture media on two sites. Therefore, each treatment is equivalent to four repetitions. Areca pollen grains were cultured on culture media with different boric acid concentrations for $150 \mathrm{~min}$ to 180 min. The pollen samples comprised approximately 30 uniformly dispersed pollen grains. Table 1 lists the observation results, which were initially transformed using

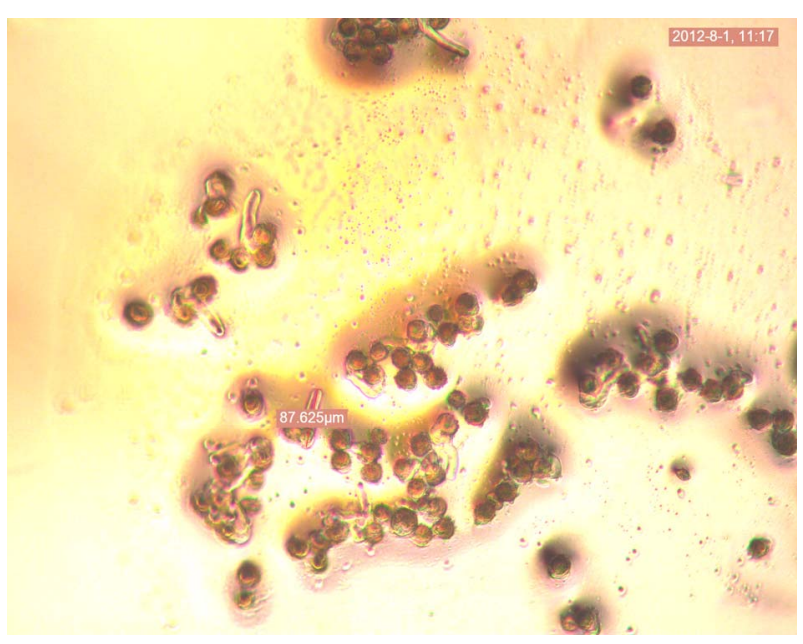

Figure 1. The initial germination stage.

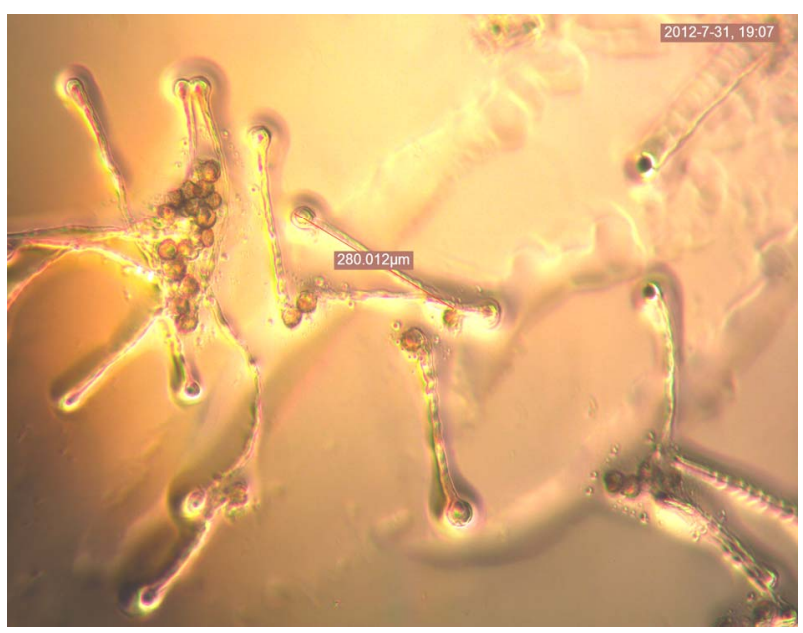

Figure 2. The mature growth stage. 
the arcsine square root in DPS statistical software. Then, variance analysis and multiple comparisons were performed on the transformed results using Duncan's new multiple range test. Finally, the findings were mapped using MS Excel (Figure 3).

Table 1 and Figure 1 show that areca pollen exhibits a low germination rate in the medium without boric acid. The addition of $0.1 \mathrm{~g} / \mathrm{L}$ boric acid significantly increases the pollen germination rate. Although the germination rate increases at boric acid concentrations of $0.1,0.2$, and $0.4 \mathrm{~g} / \mathrm{L}$, the difference does not reach a level of significance. As boric acid concentration increases to $0.6 \mathrm{~g} / \mathrm{L}$, germination rate decreases, but this reduction does not reach a level of significance. As boric acid concentration

Table 1. Effects of different boric acid concentrations on areca pollen germination.

\begin{tabular}{cccc}
\hline $\begin{array}{c}\text { Boric acid } \\
\text { concentration }(\mathrm{g} / \mathrm{L})\end{array}$ & $\begin{array}{c}\text { Germination } \\
\text { rate }(\%)\end{array}$ & $\begin{array}{c}5 \% \\
\text { (significant) }\end{array}$ & $\begin{array}{c}1 \% \text { (highly } \\
\text { significant) }\end{array}$ \\
\hline 0 & $3.2 \pm 0.81$ & $\mathrm{f}$ & $\mathrm{E}$ \\
0.1 & $90.7 \pm 6.13$ & $\mathrm{a}$ & $\mathrm{A}$ \\
0.2 & $90.3 \pm 3.66$ & $\mathrm{a}$ & $\mathrm{A}$ \\
0.4 & $92.9 \pm 2.57$ & $\mathrm{a}$ & $\mathrm{A}$ \\
0.6 & $91.3 \pm 4.65$ & $\mathrm{a}$ & $\mathrm{A}$ \\
0.8 & $84.7 \pm 4.08$ & $\mathrm{~b}$ & $\mathrm{~B}$ \\
1.0 & $80.3 \pm 5.97$ & $\mathrm{c}$ & $\mathrm{B}$ \\
2.0 & $69.5 \pm 3.13$ & $\mathrm{~d}$ & $\mathrm{C}$ \\
3.0 & $10.4 \pm 1.48$ & $\mathrm{e}$ & $\mathrm{D}$ \\
4.0 & $4.3 \pm 1.42$ & $\mathrm{f}$ & $\mathrm{E}$ \\
5.0 & $0 \pm 0.00$ & $\mathrm{~g}$ & $\mathrm{~F}$ \\
\hline
\end{tabular}

Note: 1 . The lower-case letters represent $5 \%$ significant level. 2 . The capital letters represent $1 \%$ significant level.

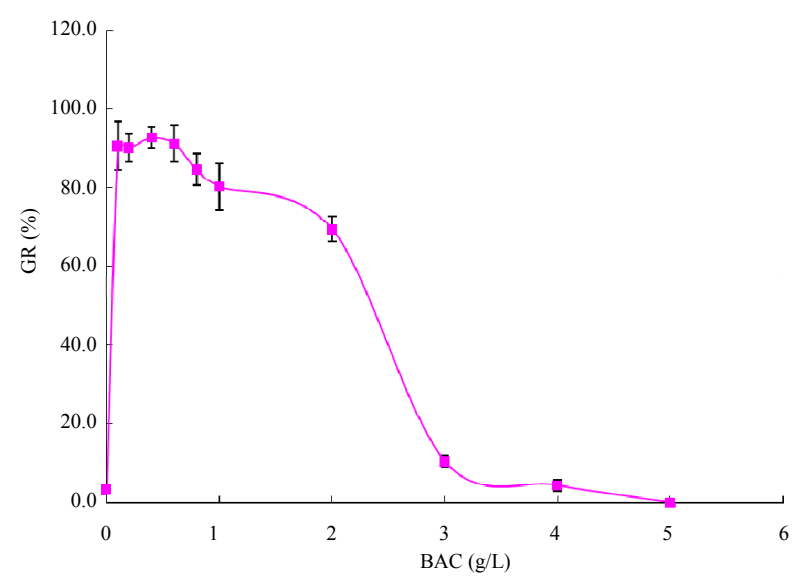

Figure 3. Effects of different boric acid concentrations on areca pollen germination rate. BAC: Boric acid concentration (g/L); GR: Germination rate $(\%)$. increases to $0.8 \mathrm{~g} / \mathrm{L}$, germination rate decreases to a highly significant level. At concentrations of 0.8 and 1.0 $\mathrm{g} / \mathrm{L}$, germination rate decreases to a significant level. At $1.0 \mathrm{~g} / \mathrm{L}$ to $5.0 \mathrm{~g} / \mathrm{L}$, the previous difference in each gradient reaches a high level of significance. Figure 1 shows that as concentration increases to $3.0 \mathrm{~g} / \mathrm{L}$, germination rate sharply declines. A $5.0 \mathrm{~g} / \mathrm{L}$ concentration completely inhibits areca pollen germination.

\subsubsection{Effects of Boric Acid Concentration on the Growth of Areca Pollen}

The preparation of culture media with different concentrations of boric acid and the setup of glass slides were in full accordance with the conditions described in Section 2.1.1. Observation proceeded at $180 \mathrm{~min}$ to $210 \mathrm{~min}$, during which the length of when the areca pollen tube was basically stable. Stretched areca pollen tubes of medium growth length were selected from each culture medium and measured. Variance analysis and multiple comparison were carried out on the results using DPS statistical software and Duncan's new multiple range test (Table 2),

Table 2. Effects of different boric acid concentrations on areca pollen length.

\begin{tabular}{cccc}
\hline $\begin{array}{c}\text { Boric acid } \\
\text { concentration }(\mathrm{g} / \mathrm{L})\end{array}$ & $\begin{array}{c}\text { Average length of } \\
\text { areca pollen tube }(\mu \mathrm{m})\end{array}$ & $\begin{array}{c}5 \% \\
(\text { significant })\end{array}$ & $\begin{array}{c}1 \% \text { (highly } \\
\text { significant) }\end{array}$ \\
\hline 0 & $38.3 \pm 1.53$ & $\mathrm{~h}$ & $\mathrm{G}$ \\
0.1 & $147.5 \pm 12.30$ & $\mathrm{e}$ & $\mathrm{E}$ \\
0.2 & $225.3 \pm 23.40$ & $\mathrm{~b}$ & $\mathrm{BC}$ \\
0.4 & $275.2 \pm 10.71$ & $\mathrm{a}$ & $\mathrm{A}$ \\
0.6 & $243.4 \pm 15.06$ & $\mathrm{~b}$ & $\mathrm{~B}$ \\
0.8 & $202.5 \pm 22.42$ & $\mathrm{c}$ & $\mathrm{CD}$ \\
1.0 & $179.0 \pm 14.16$ & $\mathrm{~d}$ & $\mathrm{D}$ \\
2.0 & $134.9 \pm 14.41$ & $\mathrm{e}$ & $\mathrm{E}$ \\
3.0 & $87.6 \pm 4.52$ & $\mathrm{f}$ & $\mathrm{F}$ \\
4.0 & $58.8 \pm 9.91$ & $\mathrm{~g}$ & $\mathrm{G}$ \\
5.0 & $0 \pm 0.00$ & $\mathrm{i}$ & $\mathrm{H}$ \\
\hline
\end{tabular}

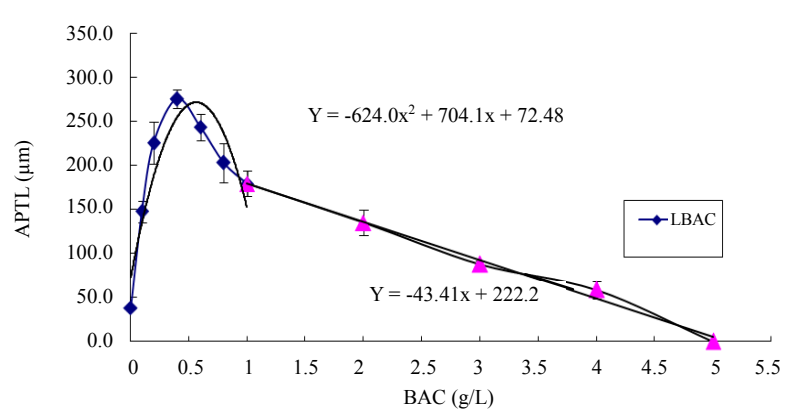

Figure 4. Effects of different boric acid concentrations on areca pollen length. APTL: Areca pollen tube length; HBAC: High boric acid concentration; LBAC: Low boric acid concentration. 
respectively. MS Excel was used to map the results (Figure 4).

As shown in Table 2, the differences among various treatments reach a significant level, and the differences among most treatments reach an extremely significant level. As shown in the scatter diagram in Figure 2, low (less than $1.0 \mathrm{~g} / \mathrm{L}$ ) and high boric acid concentrations (higher than $1.0 \mathrm{~g} / \mathrm{L}$ ) impose different effects on the length of areca pollen tube. The effect of low boric acid concentration on length follows a parabolic law. The scattered points were regressively analyzed using a quadratic polynomial; $\mathrm{R}^{2}=0.8444^{* *}(0.01, \mathrm{n}-3=4)$ was obtained. The effect of high boric acid concentration on the length of areca pollen tube exhibits a linear law. The scatter points were fitted using a linear regressive equation; we obtained $\mathrm{R}^{2}=0.9922^{* *}(0.01, \mathrm{n}-2=3)$. By calculation, the apex of the parabola of low boric acid concentration is $(\mathrm{X}=0.5642, \mathrm{Y}=271.10)$. The optimal boric acid concentration is, theoretically, $0.56 \mathrm{~g} / \mathrm{L}$.

\subsection{Effects of Culture Medium Type and Sucrose Concentration on Areca Pollen Germination Rate}

This section compares the germination rates of areca pollen grains cultured in solid and liquid culture media. The solid culture medium comprises different concentrations of sucrose, $5 \mathrm{~g} / \mathrm{L}$ agar, and different concentrations of boric acid. Sucrose concentrations were $0,20,40,60$, $80,90,100,120,140$, and $160 \mathrm{~g} / \mathrm{L}$. The formula of the liquid culture medium excludes agar, but the other compositions are the same as those of the solid culture medium. Figure 5 shows the observation data and culture media in different forms. The two culture media present large differences under the same sucrose concentration. The t-test comparison using DPS software shows that the germination rate in the solid culture medium is significantly higher than that in the liquid culture medium. This

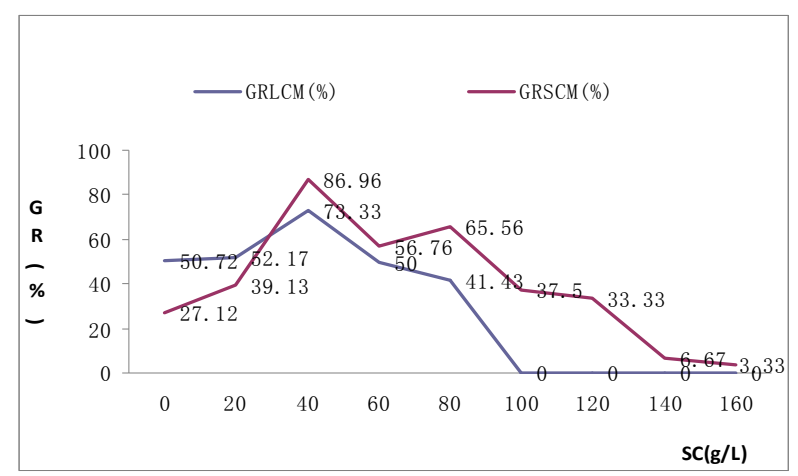

Figure 5. Effects of culture medium and sucrose concentration on areca pollen germination rate. GRLCM: Germination rate in liquid culture medium; GRSCM: Germination rate in solid culture medium; SC: Sucrose concentration; GR: Germination rate. difference reaches significance $(\mathrm{t}=2.578, \mathrm{df}=26,0.01<$ $\mathrm{P}=0.016<0.05)$. The areca pollen grains in both culture media display the maximum germination rate at a sucrose concentration of $40 \mathrm{~g} / \mathrm{L}$. However, the germination rate in the solid culture medium is $13.63 \%$ higher than that in the liquid culture medium. In the solid culture medium, the germination rate is $3.33 \%$ to $6.67 \%$ at a sucrose concentration of $140 \mathrm{~g} / \mathrm{L}$ to $160 \mathrm{~g} / \mathrm{L}$. In the liquid culture medium, the germination rate decreases from $41.43 \%$ to $0 \%$ as sucrose concentration increases from 80 $\mathrm{g} / \mathrm{L}$ to $100 \mathrm{~g} / \mathrm{L}$.

\subsection{Effects of Incubation Time on the Growth of Areca Pollen Tube}

Another culture medium was composed of $0.4 \mathrm{~g} / \mathrm{L}$ boric acid, $40 \mathrm{~g} / \mathrm{L}$ sucrose, and $5 \mathrm{~g} / \mathrm{L}$ agar. Two culture medium drops were placed on two glass slides to achieve four repetitions. Areca pollen grains were cultured in these media for $40 \mathrm{~min}$. Then, we selected one good view from each slide and one fixed areca pollen tube from these views for observation at different time points. The lengths of the areca pollen tubes were measured every 30 min up to a culture period of $340 \mathrm{~min}$. We observed parts of the areca pollen tube, which present haustorium fractures at the top section of the tubes at different times. On the basis of the measured data and using MS Excel, we determined and plotted the relationship between culture time and average areca pollen tube length (Figure 6).

The scatter points in Figure $\mathbf{4}$ were curve fitted using the logistic regression statistical method in ORIGIN 8.0. The regression equation is

$$
\mathrm{Y}=275.88 /\left(1+\mathrm{e}^{-0.0295(\mathrm{x}-91.69)}\right)
$$

$\left(\mathrm{R}^{2}=0.9925 ; \mathrm{P}<0.01\right)$. As indicated in this equation,

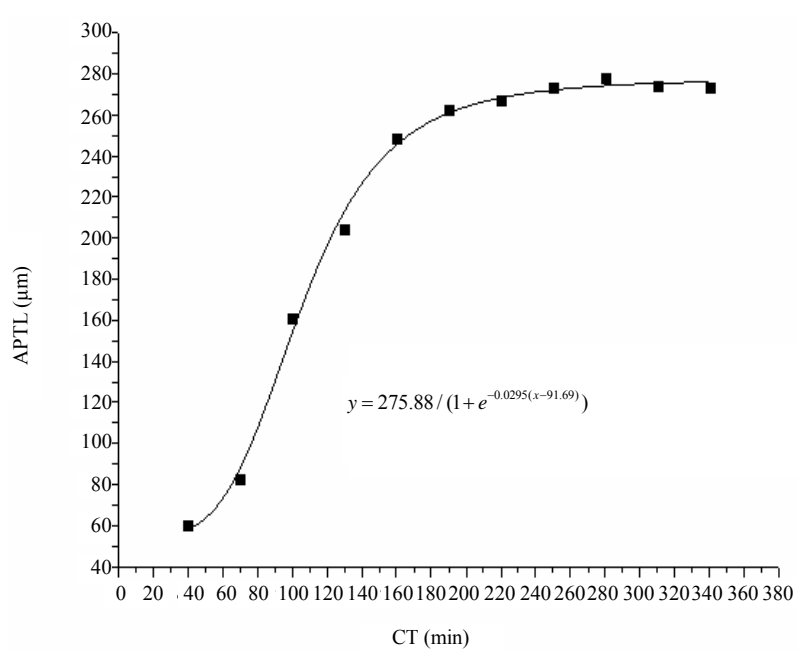

Figure 6. Relationship between culture period with areca pollen tube length. CT: Culture time; APTL: Areca pollen tube length. 
areca pollen tube can grow to a theoretical maximum length of $275.88 \mu \mathrm{m}$. In addition, it can, theoretically, grow to $10 \%, 25 \%, 50 \%, 75 \%$, and $90 \%$ of the maximum length at $17.21,54.45,91.69,128.93$, and $166.18 \mathrm{~min}$, respectively.

\section{Discussion}

\subsection{Boric Acid Is Key to Areca Pollen Germination and Growth}

The effects of boric acid on the germination and growth of pollen were reported [6,7]. Boron can affect the ductility of cell walls by changing the polysaccharide reticular formation of these walls. It can also promote pollen germination and help the pollen tube rapidly enter the ovary. Thus, boron is favorable for fertilization and seed formation. Anther and filament atrophy occur in borondeficient conditions, a phenomenon that causes difficulties in the formation of pollen tubes, thereby hindering fertilization [8]. Teng et al. found that the germination and growth of pollen can be considerably improved through the addition of boric acid of appropriate concentrations. In vitro pollen germination would be hindered if boric acid are not added or if high concentrations of boric acid are added.

In our studies, the germination rate of areca pollen is extremely low value in culture media without boric acid. The addition of $0.1 \mathrm{~g} / \mathrm{L}$ boric acid significantly improves germination rate. The germination rate of areca pollen is very high at boric acid concentrations of $0.1,0.2,0.4$, and $0.6 \mathrm{~g} / \mathrm{L}$. It reaches its maximum at $0.4 \mathrm{~g} / \mathrm{L}$. However, the differences among the four concentrations are nonsignificant. When boric acid concentration reaches 0.8 $\mathrm{g} / \mathrm{L}$, areca pollen germination rate significantly decreases. Moreover, the rate visibly decreases under boric acid concentrations of 1.0, 2.0, 3.0, 4.0, and $5.0 \mathrm{~g} / \mathrm{L}$. The germination rate, particularly at a concentration of $2.0 \mathrm{~g} / \mathrm{L}$ to $3.0 \mathrm{~g} / \mathrm{L}$, most substantially decreases; that is, from $69.5 \%$ to $10.4 \%$. When boric acid concentration reaches $5.0 \mathrm{~g} / \mathrm{L}$, areca pollen germination is completely suppressed. Different boric acid concentrations also impose significant effects on the growth of areca pollen tube. At a boric acid concentration of $0 \mathrm{~g} / \mathrm{L}$ to $0.56 \mathrm{~g} / \mathrm{L}$, pollen tube length gradually grow in a parabolic manner with increasing boric acid concentration. At a boric acid concentration of $0.56 \mathrm{~g} / \mathrm{L}$ to $1.0 \mathrm{~g} / \mathrm{L}$, pollen tube length gradually decreases in a parabolic manner with increasing boric acid concentration. At $1.0 \mathrm{~g} / \mathrm{L}$ to $5.0 \mathrm{~g} / \mathrm{L}$, pollen tube length gradually decreased in a linear manner with increasing boric acid concentration. The comprehensive analysis of the effects of boric acid concentration on areca pollen germination rate and areca pollen tube growth indicates that the optimum boric acid concentration for areca pollen culture medium is $0.4 \mathrm{~g} / \mathrm{L}$ to $0.6 \mathrm{~g} / \mathrm{L}$.

\subsection{Appropriate Sucrose Concentrations Are Essential for Areca Pollen Germination and Growth}

Sugar can regulate the osmotic potential in pollen tube growth. It is also a source of nutrients and energy [9]. At suitable sucrose concentrations, the balance between the internal and external osmotic pressures of pollen can be maintained, thereby preserving the normal vitality of pollen. A suitable sucrose concentration is conducive to pollen germination [10]. Stanli [11] indicated that the conditions provided by in vitro germination closely resembles those during pollen germination in stigma. Thus, the pollen viability obtained by in vitro germination is also close to that obtained by pollen germination in stigma. Zhao [12,13], Li [14], and Peng et al. [15] investigated the suitability of solid or liquid culture medium. Peng et al. found that Xanthoceras sorbifolia presents a high and low germination rate in solid and liquid culture media, respectively.

In the current work, different gradients of sucrose concentrations exert strong effects on areca pollen germination. Areca pollen shows the highest germination rate at a sucrose concentration of $40 \mathrm{~g} / \mathrm{L}$ in both the solid and liquid culture media. At low sucrose concentrations, the germination rate is higher in the liquid than in the solid culture medium. At high sucrose concentrations, the germination rate was higher in the solid than in the liquid culture medium. Generally, the germination rate in the solid culture medium is higher than that in the liquid culture medium. In the liquid culture medium, the germination rate is $0 \%$ at a sucrose concentration of $100 \mathrm{~g} / \mathrm{L}$. In the solid culture medium, the germination rate is 3.33\% at a sucrose concentration of $160 \mathrm{~g} / \mathrm{L}$. This finding may be attributed to the fact that when the areca pollen grains are immersed in liquid medium of high sucrose concentrations, the extremely unbalanced osmotic pressure in pollen grains inhibits the germination of areca pollen. In addition, the total areca pollen germination rate is lower than the areca pollen germination rate in previous boron effect tests. This difference may be attributed to the fact that the pollen grains used in the sucrose effect tests were collected during the late areca flowering phase; thus, poor-quality areca pollen was obtained.

\subsection{Effects of Different Culture Periods on the Growth of Areca Pollen Tube}

This study also shows the principle by which areca pollen tube grows at different culture periods. The relationship between the growth in length of areca pollen tube and culture time presents an S-growth curve law. The logistic regression curve analysis reveals a high fitting coefficient. As indicated by logistic regression analysis, the areca pollen tubes rapidly grows $50 \mathrm{~min}$ to $60 \mathrm{~min}$ 
post-culture. Then, it rapidly grows at a culture time of $90 \mathrm{~min}$ to $100 \mathrm{~min}$. The growth rate of areca pollen tube slows down at a culture time of $150 \mathrm{~min}$. The length exceeds $90 \%$ of the maximum value and tends to stabilize at a culture period of $170 \mathrm{~min}$.

\subsection{Conclusion}

Boric acid can significantly promote the germination and growth of areca pollen. Growth promotion is most notable at a boric acid concentration of $0.4 \mathrm{~g} / \mathrm{L}$ to $0.6 \mathrm{~g} / \mathrm{L}$. Sucrose imposes significant effects on areca pollen germination. The effects are most obvious at a sucrose concentration of $40 \mathrm{~g} / \mathrm{L}$. The solid culture medium with agar presents better effects than does the liquid culture medium without agar. The optimum culture medium for developing areca pollen comprises $5 \mathrm{~g} / \mathrm{L}$ agar, $0.4 \mathrm{~g} / \mathrm{L}$ to $0.6 \mathrm{~g} / \mathrm{L}$ boric acid, and $40 \mathrm{~g} / \mathrm{L}$ sucrose. Areca pollen grows most rapidly approximately $1.5 \mathrm{~h}$ after in vitro cultivation, and growth stabilizes at approximately $3 \mathrm{~h}$. This finding serves as solid theoretical basis for developing fruit-protecting reagents with boric acid and sucrose, which can be sprayed in areca palm plantations. Moreover, the use of these substances is significant in controlling flower and fruit drop in areca palm.

\section{Acknowledgements}

This research was supported by the agricultural technology promotion project by the Chinese Ministry of Agriculture (Grant no. 13RZNJ-10) and the protection project of tropical crops germplasm resources by the Chinese Ministry of Agriculture (protection project of arecanut germplasm resources; Grant No. 13RZZR-17).

\section{REFERENCES}

[1] R. C. Pan, X. J. Wang and N. H. Li, "Plant Physiology," Higher Education Press, Beijing, 2008, p. 36.

[2] T. Zhang and M. Huang, "The Effect of Sucrose and PEG on the in Vitro Germination of Camellia Pollen," Northern Horticulture, Vol. 1, 2009, pp. 101-102.
[3] X. P. Liu, J. X. Tu and B. Y. Chen, "Research Progress in Yellow-Seeded Rapeseed," Chinese Journal of Oil Crop Sciences, Vol. 27, No. 2, 2005, pp. 87-91.

[4] Y. H. Yang, Y. S. Xia and C. W. Du, "The Difference on the Boron Demand in the Vegetative Growth of the Rape Cultivars with Different Boron Efficiencies," Chinese Journal of Oil Crop, Vol. 27, No. 1, 2005, pp. 69-72.

[5] Z. L. Zhang, J. N. Li and Z. L. Tang, "The Regulation of Environmental Conditions on Rape Quality," Chinese Agricultural Science Bulletin, Vol. 2, 2006, pp. 124-129.

[6] C. C. Teng and Y. C. Zhang, "The Influence of Boric Acid and Sucrose on the in Vitro Germination of Potato Pollen," Seed, Vol. 28, No. 2, 2009, pp. 15-20.

[7] G. J. Liang, G. P. Huang, L. Deng, et al., "Effect of Boron, Sugar, Calcium and DA-6 on the Growth of Loquat Pollen Tube," Journal of Zhaoqing University, Vol. 32, No. 2, 2011, pp. 50-56.

[8] Zhejiang Agricultural University, "Plant Nutrition and Fertilizer Science," Vol. 10, 1992, p. 143.

[9] G. S. Zhang, "Effect of Boron and Sugar on the Growth of Cucurbita Maxima Pollen Tube," Journal of Heze University, Vol. 22, No. 2, 2000, pp. 54-55.

[10] T. Zhang and M. Huang, "The Effect of Sucrose and PEG on the in Vitro Pollen Germination of Camellia," Northern Horticulture, Vol. 1, 2009, pp. 101-102.

[11] R. G. Stenli and H. F. Linskens, "Pollen: Biology Biochemistry Management," Springer-Verlag, Berlin, Heidelberg, New York, 1974, p. 37.

[12] H. B. Zhao, W. M. Fang and F. D. Chen, "Pollen Germination in Vitro and Pollen Tube Growth of Plum," GUIHAIA, No. 3, 2007, pp. 393-396.

[13] H. B. Zhao, F. D. Chen and W. M. Fang, "Pollen Germination in Vitro of Chrysanthemum Cultivars with Small Inflorescences and Several Species of Dendranthema," Journal of Nanjing Agricultural University, Vol. 28, No. 2, 2005, pp. 22-27.

[14] Z. N. Li, G. F. Liu, C. L. Luo, et al., "The Viability and Storage Capacity of Platanus orientalis Pollen," Wuhan Botanical Research, Vol. 24, No. 1, 2006, pp. 54-57.

[15] W. X. Peng, F. L. Li and W. L. Yang, "Determination and Comparison of the Viability of Various Xanthoceras sorbifolia Pollen," Hebei Journal of Forestry and Orchard Research, Vol. 14, No. 1, 1999, pp. 51-53. 